\section{Genomic characterization of spleens in patients with myelofibrosis}

Despite the central role of splenomegaly in the pathophysiology of myelofibrosis (MF), evidence regarding the genomic landscape within these spleens is limited. In a study reporting the splenic cytogenetics of 92 patients with MF who underwent splenectomy, 52 (56\%) had abnormal splenic karyotypes. A comparison between spleen and bone marrow (BM) karyotypes was available in 68 patients, with concordant results in $85 \%$ and discordant in $15 \%$. In 9 out of the 10 patients with discordant findings, additional abnormalities occurred in the spleens. ${ }^{1}$ Subsequent studies showed presence of $J A K 2 \mathrm{~V} 617 \mathrm{~F}$ in the spleens of MF patients, and reported rare cases of $J A K 2 \mathrm{~V} 617 \mathrm{~F}$ occurring in the spleen but not the BM. ${ }^{2,3}$ Since the publication of these studies, technologies that provide significantly higher resolution of genomic alterations have emerged. To the best of our knowledge, chromosomal microarrays and next generation sequencing (NGS) have not been applied to study MF spleens. We performed conventional cytogenetics, $\mathrm{FISH}$, array-comparative genomic hybridization with single nucleotide polymorphisms microarray (aCGH+SNP), and NGS on spleen cells from 14 patients with MF who had undergone splenectomy from 2011 until 2015, and assessed the degree of genomic diversity. We hypothesized that specific genomic aberrations might be unique to the spleen, and can potentially contribute to disease progression.

We previously reported the clinical characteristics and post-splenectomy outcomes of 12 of the 14 patients. ${ }^{4}$ Our cohort included patients with primary MF and post-polycythemia vera or post-essential thrombocythemia MF who fulfilled the 2008 WHO diagnostic criteria. ${ }^{5}$ Median age was 65 years (range 51-79). Indications for splenectomy were refractory cytopenias in 10 cases, symptomatic splenomegaly not responding to ruxolitinib in 2 , and preparation for stem-cell transplantation in 2. Median post-splenectomy survival was 14 months but ranged widely from 34 days to over four years. Following ethical approval, single-cell suspensions from spleen specimens were prepared as previously described. ${ }^{6} \mathrm{BM}$ or peripheral blood (PB) samples were collected within two months prior to and/or following splenectomy. The genomic studies are summarized in Table 1.

Metaphase cytogenetics and interphase-FISH were performed using standard methodology. Cytogenetic results were concordant between spleens and $\mathrm{BM} / \mathrm{PB}$ in 12 $(86 \%)$ patients, including 7 with a normal karyotype and 5 with abnormal karyotypes. Chromosomal abnormalities occurred at comparable frequencies in both tissue specimens. Discordant findings were observed in $2(14 \%)$ patients (ns. SP01 and SP09), both harboring a complex karyotype in which the spleen contained additional abnormalities that were not detected in the PB. In patient SP01, who had pentasomy-21 in spleen only, trisomy-21 was subsequently observed in a post-splenectomy PB karyotype. In another patient (n. SP02), who at the time of splenectomy had a concordant normal karyotype, del(20q) was detected in a single (non-clonal) spleen metaphase cell. This patient developed a dominant $\operatorname{del}(20 \mathrm{q})$ clone (85\% of metaphase cells) in a BM study performed 2.5 years post splenectomy, suggesting that this clone originated in the spleen. Since the spleen cells from each patient always contained at least the same abnormalities as the $\mathrm{BM} / \mathrm{PB}$, it seems likely that $\mathrm{MF}$ originated in the $\mathrm{BM}$ and later migrated to the spleen.
However, the additional abnormalities detected in the spleens suggest that clonal evolution can occur within the MF spleen and that these cells then re-enter the $\mathrm{BM} / \mathrm{PB}$ post splenectomy. This hypothesis is supported by evidence of leukemic transformation arising in the spleens of patients with myeloproliferative neoplasms (MPN). ${ }^{7}$ Other explanations, such as the emergence of previously undetectable clones in the $\mathrm{BM}$, are also possible.

Array-CGH+SNP was performed on genomic DNA extracted from spleen cells available from 13 patients, using GenetiSure Unrestricted-2X400K array (Agilent Technologies, Santa Clara, CA, USA). This platform can identify copy-number alterations (CNAs) and copy-neutral loss of heterozygosity (CN-LOH) involving 2,300 cancer-related genes with an average of 6 probes per exon, affording a resolution of $<500 \mathrm{bp}$ for CNAs and $2.5 \mathrm{Mb}$ for $\mathrm{CN}-\mathrm{LOH}$. CNAs were called if they were $>500 \mathrm{bp}$ and contained a minimum of five probes. Regions of CN-LOH were called if they were $\geq 5 \mathrm{Mb}$. For each CNA detected we reviewed the Database of Genomic Variants (DGV) (http://dgv.tcag.ca/dgv) to exclude normal copy-number variants.

Array-CGH+SNP revealed gain of 168 regions, loss of 43 regions and 37 regions of $\mathrm{CN}-\mathrm{LOH}$ (Figure 1A). Genomic alterations were detected in all patients, including those with a normal karyotype. In patient n. SP01, aCGH+SNP revealed chromothripsis that was cytogenetically described as $\operatorname{der}(5 \mathrm{q})$, included 78 alterations, and correlated with a TP53 mutation as shown by NGS. Chromothripsis was recently linked to TP53 alterations in acute myeloid leukemia with complex karyotypes. When patient n. SP01 was excluded from the analysis, the mean number of genomic alterations per patient was 14 (range 7-20) including 9.4 gains, 2.1 losses, and 2.6 $\mathrm{CN}-\mathrm{LOH}$. These frequencies are higher than previously reported in array studies of advanced MF, likely due to the higher resolution of this platform. Array analysis confirmed all cytogenetic abnormalities except for balanced $\mathrm{t}(8 ; 12)$ in SP05, and $\mathrm{del}(20 \mathrm{q})$ in $5 \%$ of metaphase cells (below the sensitivity of array testing) in patient $\mathrm{n}$. SP14. However in patient n. SP14, aCGH+SNP identified deletion of ETV6 (12p13.2), confirmed by FISH in more than $60 \%$ of both spleen and PB cells, that was not detected by metaphase cytogenetics. Additional clinically relevant abnormalities revealed solely by array were regions of $\mathrm{CN}-\mathrm{LOH}$ involving mutated genes, such as CN-LOH of $9 \mathrm{p}$ in $4 J A K 2$-mutated patients.

While many of the novel genomic alterations detected by array occurred in single cases, eight regions of gain were each identified in a remarkably high proportion of patients. Four of these recurrent regions occurred within RHOC (1p13.2), NCOR2 (12q24.31), RASA3 (13q34), and TAF15 (17q12), genes reported in association with myeloid malignancies ${ }^{9-12}$ (Table 2). RHOC encodes a GTPase up-regulated in numerous solid cancers, and was previously implicated in leukemogenesis. ${ }^{9}$ NCOR2 encodes SMRT, a member of a protein complex that can modify chromatin structure or bind to nuclear receptors to repress transcription. Remarkably, mice carrying inactivating mutations in SMRT developed a syndrome resembling $M F$ that included $B M$ fibrosis and splenomegaly. SMRT inactivation resulted in reduced repression of the retinoic acid receptor, leading to increased cytokine gene expression, particularly thrombopoietin and TGF $\beta$, resembling pathological pathways that occur in MF patients. ${ }^{10} R A S A 3$ encodes for a negative regulator of the RAS and RAP1 pathways, and inhibitor of RAP1-dependent platelet activation. Inactivation of 
Table 1. Genomic characterization of spleens in 14 patients with myelofibrosis.

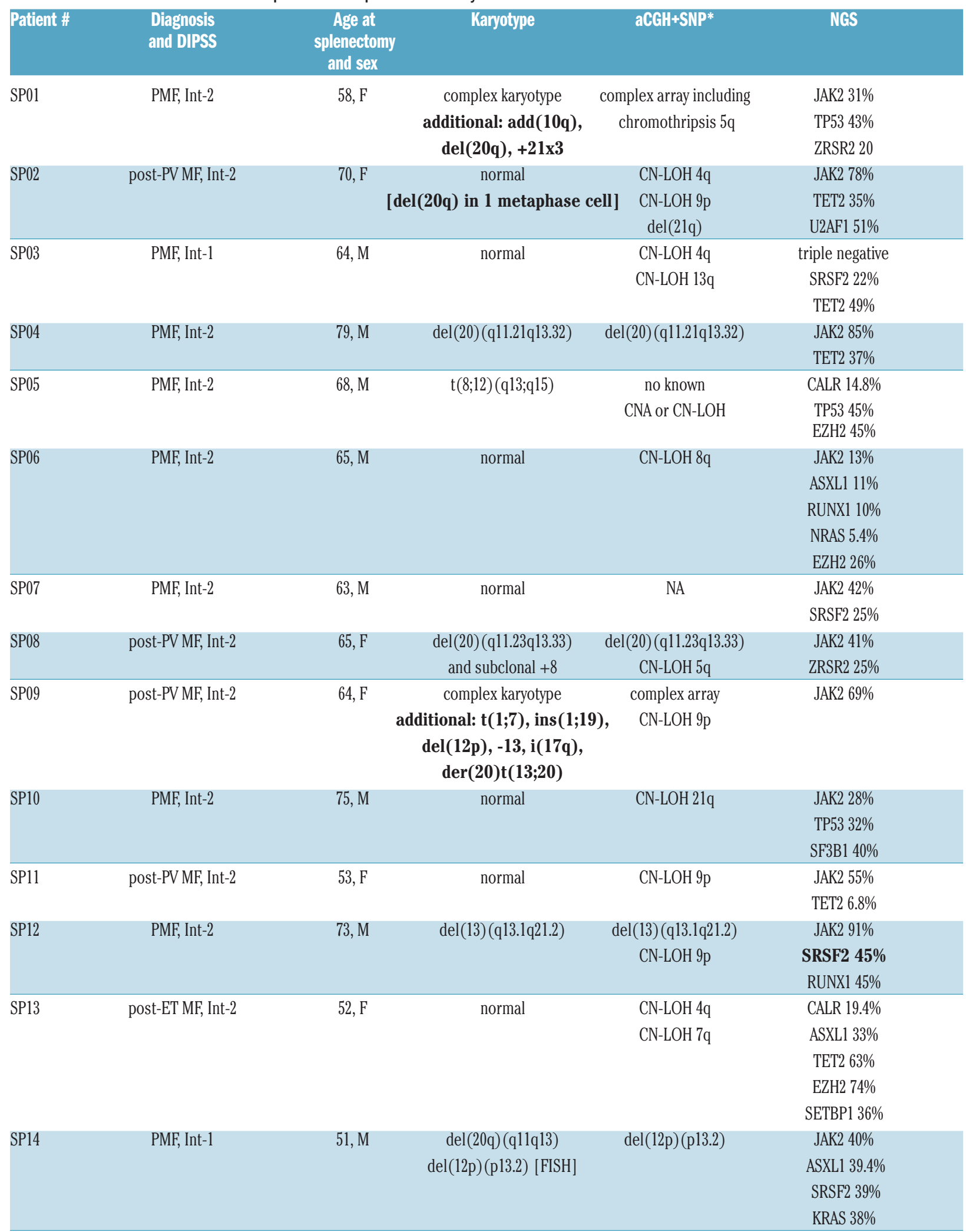

DIPSS: Dynamic International Prognostic Scoring System; aCGH+SNP: array comparative genomic hybridization and single nucleotide polymorphisms microarray; NGS: next generation sequencing; PMF: primary myelofibrosis; Post-PV MF: post-polycythemia vera myelofibrosis; Post-ET MF: post-essential thrombocythemia myelofibrosis; CNA: copy number alteration; $\mathrm{CN}-\mathrm{LOH}$ : copy-neutral loss of heterozygosity; F: female; M: male; NA: not available. Text appearing in bold indicates cases with abnormalities found in the spleen but not the peripheral blood or bone marrow. * Only selected regions known to be recurrent in myeloid malignancies are shown. For complete results of our aCGH+SNP analysis, see Figure 1. 
RASA3 in mouse hematopoietic cells led to megakaryocyte dysplasia and resulted in cytopenias, BM fibrosis, splenomegaly and decreased survival. ${ }^{11}$ TAF15 is involved in the fusion-gene product of a rare translocation reported in acute leukemia. ${ }^{12}$

In validation analyses, the recurrent regions were observed in a cohort of 173 patients with myeloid malignancies (Table 2), albeit at lower frequencies, but not in any of 42 individuals without disease. Gain within NCOR 2 was reported in the DGV in 2 individuals $(<1 \%)$, but was not excluded from our analysis due to its high frequency in our cohort. Furthermore, 3 patients with this gain harbored mutations in NCOR2 by NGS, located distal from the region of gain. Focal gains or duplications occurring in either coding or non-coding sequences within a gene can lead to its decreased or increased expression or to aberrant function. ${ }^{13}$ From these studies, we suggest that these novel abnormalities might play a role in disease progression.

Simultaneous aCGH+SNP was performed on matched spleen and $\mathrm{PB}$ specimens from 4 patients (Figure 1B). Abnormalities of known clinical significance occurred in both spleen and PB. However, multiple discordant CNAs and regions of $\mathrm{CN}-\mathrm{LOH}$ in all 4 patients were observed, with a higher number of abnormalities occurring in the spleens.

To complete the genomic characterization of $\mathrm{MF}$ spleens, high-throughput sequencing with a targeted

A

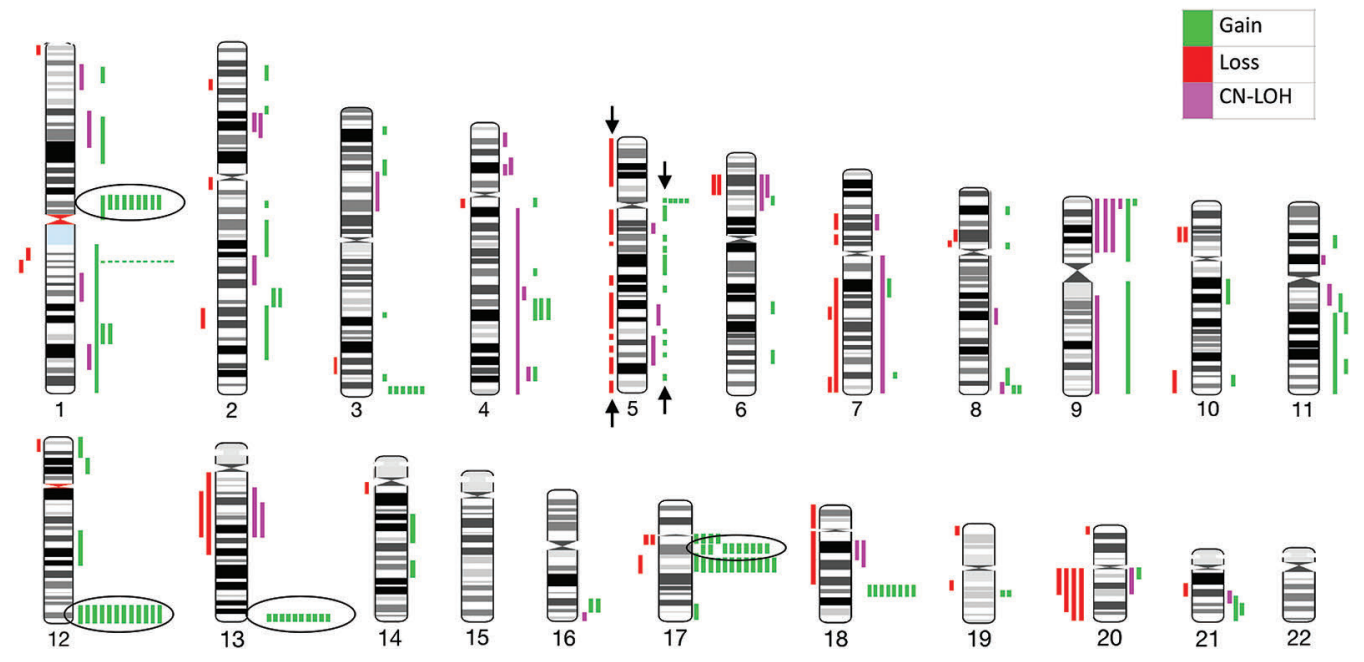

B

\begin{tabular}{|c|c|c|c|}
\hline \multicolumn{4}{|c|}{ SP11 } \\
\hline Chromosome Band & Type & SP & PB \\
\hline $1 \mathrm{p} 31.1$ & $\mathrm{LOH}$ & & \\
\hline $1 \mathrm{p} 13.2$ & GAIN & & \\
\hline $1 \mathrm{q} 21.2$ & Loss & & \\
\hline 1q22 & GAIN & & \\
\hline $2 q 31.1$ & GAIN & & \\
\hline 3929 & GAIN & & \\
\hline $8 q 11.21$ & Loss & & \\
\hline 9p24.3 - p13.1 & LOH & & \\
\hline $11 \mathrm{q} 23.3$ & Loss & & \\
\hline $12 q 24.31$ & GAIN & & \\
\hline $12 q 24.33$ & GAIN & & \\
\hline $13 q 34$ & GAIN & & \\
\hline $14 q 11.2$ & LOSS & & \\
\hline $17 p 13.2$ & LOSS & & \\
\hline $17 q 11.2$ & GAIN & & \\
\hline $17 q 12$ & GAIN & & \\
\hline $17 q 21.2$ & LOSS & & \\
\hline $17 q 21.31$ & GAIN & & \\
\hline $18 q 11.1$ & LOSS & & \\
\hline $18 \mathrm{q} 12.1$ - $\mathrm{q} 12.2$ & LOH & & \\
\hline $18 q 21.31$ & GAIN & & \\
\hline $20 q 11.21$ & GAIN & & \\
\hline$x_{p 11.23}$ & GAIN & & \\
\hline
\end{tabular}

SP12

Chromosome Band Type SP PB $1 \mathrm{p} 36.12$ - $\mathrm{p35.1} \quad \mathrm{LOH}$ $1 \mathrm{q} 22$

1q23.3 $1 \mathrm{q} 24.2-\mathrm{q} 25.1$ $3 \mathrm{q} 28$ $5 \mathrm{p} 12$ $6 \mathrm{p} 22.3$ $6 \mathrm{p} 22.3$
$7 \mathrm{p} 22.3$
$7 \mathrm{p} 14.2-\mathrm{p} 14$ $7 \mathrm{p} 22.3$ 7p14.2- $\mathrm{p}$ 9p11.23 $24.3-\mathrm{p} 24.1$ 11q23.2 11q23.2 12q24.31 $13 q 12.3 \cdot q 21.2$ $17 q 11.2$ 17921.31 $18 \mathrm{q} 11.2$ 18921.33 19013.2 $20 \mathrm{p} 13$ \begin{tabular}{|l|l|}
\hline $20 p 13$ \\
\hline $21 q 22.11$ \\
\hline$X p 2233$
\end{tabular} $21 q 22.11$
Xp22.33

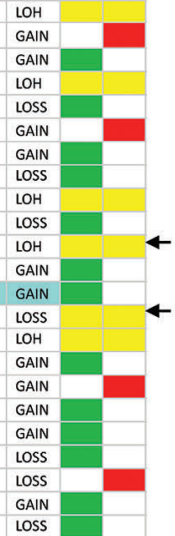

SP13

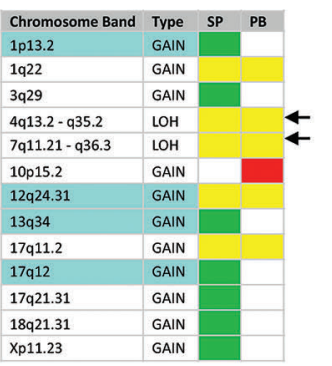

\begin{tabular}{|l|l|}
\hline Spleen only \\
\hline PB only \\
\hline Both spleen and PB \\
\hline Novel recurrent regions \\
\hline$\leftarrow \begin{array}{l}\text { Known cytogenomic } \\
\text { abnormalities }\end{array}$ \\
\hline
\end{tabular}

Figure 1. aCGH+SNP analysis. (A) Chromosome ideogram of all alterations detected in myelofibrosis (MF) spleens from 13 patients. Bars depict the physical position of the aberration but not the size. All bars shown between arrows represent genomic changes within chromothripsis of chromosome 5 in SP01. The novel recurrent regions of gains mapping to 1p13.2 (RHOC), 12q24.31 (NCOR2), 13q34 (RASA3) and 17q12 (TAF15) are shown in circles. (B) Comparison of matched spleen and peripheral blood (PB) specimens from 4 patients. Green boxes show alterations detected in spleens only; red in PB only; yellow in both tissues. Aqua highlights the novel recurrent regions of gain discussed above. Of note, in SP11 all four recurrent regions were detected in both spleen and PB, while in SP12 and SP13 most were detected only in the spleen (except for gain mapping to 12q24.31 in SP13). Arrows point to abnormalities of known clinical significance, including del(13q), del(12p13.2) involving ETV6, and CN-LOH of 9p, 4q an 7q involving JAK2, TET2 and EZH2, respectively. 
Table 2. Regions of recurrent chromosomal gains and involved genes.

\begin{tabular}{lcccccc}
$\begin{array}{l}\text { Chromosomal } \\
\text { region }\end{array}$ & $\begin{array}{c}\text { Size of } \\
\text { region (bp) }\end{array}$ & $\begin{array}{c}\text { Average } \\
\text { log2 ratio* }\end{array}$ & $\begin{array}{c}\text { Involved } \\
\text { gene }\end{array}$ & $\begin{array}{c}\text { Exon / } \\
\text { intron }\end{array}$ & $\begin{array}{c}\text { \# of patients } \\
\text { in study cohort (\%) }\end{array}$ & $\begin{array}{c}\text { \# of patients } \\
\text { in validation cohort (\%) }\end{array}$ \\
$1 \mathrm{p} 32.2$ & 786 & 0.5 & RHOC & Intron & $9(69 \%)$ & $7(4 \%)$ \\
$12 q 24.31$ & 1,895 & 1.0 & NCOR2 & Exon 49 & $12(92 \%)$ & $17(10 \%)$ \\
\hline $13 q 34$ & 1,958 & 1.3 & RASA3 & Exons 18, 17 & $11(85 \%)$ & $17(10 \%)$ \\
$17 q 12$ & 1,272 & 0.6 & TAF15 & Exons 13-15 & $9(69 \%)$ & $16(9 \%)$ \\
\hline
\end{tabular}

bp: base pairs; RHOC: Ras homolog family member C; NCOR2: Nuclear receptor corepressor 2 (also known as SMRT, Silencing Mediator of Retinoic acid and Thyroid hormone receptor); RASA3: Ras p21 protein activator 3; TAF15: TATA-box binding protein associated factor $15 .{ }^{*}$ A cutoff signal-intensity log2 ratio (between patient and reference DNA) of $\geq 0.18$ for gain or $\leq 0.18$ for loss was used. ${ }^{5}$ The validation cohort consisted of 173 patients with myeloid malignancies including acute myeloid leukemia $(n=61)$, chronic myeloid leukemia $(n=4)$, myelodysplastic syndromes $(n=61)$ and myelofibrosis $(n=47)$ of all risk categories

deep sequencing assay of 585 cancer-related genes (HemePACT) was performed as previously described. ${ }^{14}$ NGS identified an average of 2.8 oncogenic mutations per spleen. Aside from MPN driver mutations detected in 13 of the 14 patients, additional acquired mutations that have been identified in $\mathrm{MPNs}^{15}$ were detected in most patients. The most common additionally mutated genes were TET2 (in 5 patients) followed by SRSF2 (in 4 patients), and ASXL1, EZH2 and TP53 (in 3 patients). The high incidence of mutations in epigenetic modifiers and splicing factors is typical of an advanced MF patient population. NGS was used to compare matching spleen and $\mathrm{PB}$ specimens in 4 patients, harboring a total of 14 mutations. The concordance of mutations and their variant allele frequencies was strikingly high. However, an SRSF2 mutation was detected only in the spleen of SP12.

The high concordance of genomic aberrations between spleens and $\mathrm{BM} / \mathrm{PB}$, and the comparable frequency of both chromosomal abnormalities and mutation allele burden; suggest an overall similar clonal architecture existing within these tissues. In spite of the overall similarity, discordant findings were detected by each modality. In almost all of these instances, additional abnormalities were found in the patients' spleens. These findings are consistent with earlier studies comparing MF spleens to $\mathrm{BM} / \mathrm{PB}$ specimens ${ }^{1-3,6,7}$ and suggest a higher genomic complexity in the spleen.

In conclusion, these studies indicate that there are genomic differences between MF hematopoietic cells that reside in the spleen and $\mathrm{BM}$ or $\mathrm{PB}$, which might be the consequence of different tumor microenvironments. Array-CGH+SNP detected novel recurrent gains within RHOC, NCOR2, RASA3 and TAF15 that may play a role in disease pathogenesis, and these merit further investigation.

\section{Eran Zimran, ${ }^{1}$ Joseph Tripodi, ${ }^{1,2}$ Raajit Rampal, ${ }^{3}$}

Franck Rappoport, ${ }^{3,4}$ Sharon Zirkiev, ${ }^{2}$ Ronald Hoffman' ${ }^{1}$ and Vesna Najfeld ${ }^{1,2}$

${ }^{1}$ Myeloproliferative Neoplasms Research Program, Department of Hematology and Oncology, Tisch Cancer Institute, Icahn School of Medicine at Mount Sinai; ${ }^{2}$ Tumor Cytogenomics Laboratory, Department of Pathology, Icahn School of Medicine at Mount Sinai; ${ }^{3}$ Human Oncology and Pathogenesis Program, Leukemia Service, Memorial Sloan-Kettering Cancer Center and ${ }^{4}$ Center for Clinical and Translational Science, Rockefeller University, New York, NY, USA

EZ and JT contributed equally to this work.

Acknowledgments: the authors would like to thank Dr. Jane Houldsworth for her invaluable review of the manuscript, and Dr. Joseph Prchal for his generous assistance with retrieval of information.
Correspondence:vesna.najfeld@mssm.edu doi:10.3324/haematol.2018.193763

Information on authorship, contributions, and financial \& other disclosures was provided by the authors and is available with the online version of this article at www. haematologica.org.

\section{References}

1. Mesa RA, Li, CY, Schroeder G, Tefferi A. Clinical correlates of splenic histopathology and splenic karyotype in myelofibrosis with myeloid metaplasia. Blood. 2001;97(11):3665-3667.

2. Konoplev S, Hsieh PP, Chang CC, Medeiros LJ, Lin P. Janus kinase 2 V617F mutation is detectable in spleen of patients with chronic myeloproliferative diseases suggesting a malignant nature of splenic extramedullary hematopoiesis. Hum Pathol. 2007;38(12):1760-1763.

3. Hsieh PP, Olsen RJ, O'Malley DP, et al. The role of Janus Kinase 2 V617F mutation in extramedullary hematopoiesis of the spleen in neoplastic myeloid disorders. Mod Pathol. 2007;20(9):929-935.

4. Aruch D, Schwartz M, Mascarenhas J, Kremyanskaya M, Newsom C, Hoffman R. Continued role of splenectomy in the management of patients with myelofibrosis. Clin Leuk Myel Lym. 2016;16(9):e133137.

5. Thiele J, Kvasnicka HM. The 2008 WHO diagnostic criteria for polycythemia vera, essential thrombocythemia and primary myelofibrosis. Curr Hematol Malig Rep. 2009;4(1):33-40.

6. Barosi G, Vittorio R, Margherita M, et al. Spleen neoangiogenesis in patients with myelofibrosis with myeloid metaplasia. Br J Hematol. 2004;124(5):618-625.

7. Prakash S, Hoffman R, Barouk S, Wang YL, Knowles DM, Orazi A. Splenic extramedullary hematopoietic proliferation in Philadelphia chromosome-negative myeloproliferative neoplasms: heterogenous morphology and cytological composition. Mod Pathol. 2012; 25(6):815-827.

8. Rucker F, Dolnik A, Blatte TJ, et al. Chromothripsis is linked to TP53 alteration, cell cycle impairment, and dismal outcome in acute myeloid leukemia with complex karyotype. Haematologica. 2018; 103(1):e17-e20.

9. Marcucci G, Yan P, Maharry K, et al. Epigenetics meets genetics in acute myeloid leukemia: clinical impact of a novel seven-gene score. J Clin Oncol. 2014;32(6):548-556.

10. Hong SH, Dvorak-Ewell M, Stevens HY, et al. Rescue of a primary myelofibrosis model by retinoid-antagonist therapy. Proc Natl Acad Sci. 2013;110(47):18820-18825.

11. Molina-Ortiz P, Polizzi S, Ramery E, et al. Rasa3 controls megakaryocyte Rap1 activation, integrin signaling and differentiation into proplatelet. PLoS Genet. 2014;10(6):e1004420.

12. Yao L, Cen J, Pan J, et al. TAF15-ZNF384 fusion gene in childhood mixed phenotype acute leukemia. Cancer Gen. 2017;211:1-4.

13. Krijgsman O, Carvalho B, Meijer GA, Steenbergen RDM, Ylstra B. Focal chromosomal copy number aberrations in cancer - needles in a haystack. Biochim Biophys Acta. 2014;1843(11):2698-2704.

14. Kucine N, Viny AD, Rampal R, et al. Genetic analysis of five children with essential thrombocytosis identified mutations in cancer-associated genes with roles in transcriptional regulation. Haematologica. 2016;101(6):e237-e239.

15. Vainchenker W, Kralovics R. Genetic basis and molecular pathophysiology of classical myeloproliferative neoplasms. Blood. 2017; 129(6):667-679. 